\title{
Number Density Distributions of Metal Vapor in Helium Gas Tungsten Arcs
}

\author{
Y. Tsujimura, H. Terasaki, K. Yamamoto, S. Tashiro and M. Tanaka \\ Joining and Welding Research Institute, Osaka University, 11-1 Mihogaoka, Ibaraki, Osaka 576-0047 \\ e-mail: tujimura@jwri.osaka-u.ac.jp
}

\begin{abstract}
In order to investigate effects of metal vapor on the plasma state in welding process, electron temperature in helium Gas Tungsten Arc (GTA) plasma during welding was measured by using the laser scattering method. Furthermore, number density distributions of metal neutrals and ions were also measured by using the spectroscopic analysis. Our results showed that plasma during welding consisted of two plasma regions, namely, pure helium plasma region and quasi-metal plasma region.
\end{abstract}

Key words: Arc plasma, Helium, Metal vapor, Electron temperature, Number density

\section{INTRODUCTION}

It is important in the welding process to investigate effects of metal vapor on arc plasma. Contamination of metal vapor to the arc plasma would change the plasma state of the arc. It means that arc plasma during welding is quite different from pure one.

Many researchers have tried a variety of investigations on metal contaminated arc plasma. Glickstein [1] presented the result of spectroscopic measurements on a $100 \mathrm{~A}, 2 \mathrm{~mm}$ arc length, argon arc with evaporation from heated alloy 600 plate. He showed that the arc temperature results determined with a stationary molten anode are similar to the results with a cooled anode. Etemadi and Pfender [2] presented a result of spectroscopic measurements on a $150 \mathrm{~A}, 10$ $\mathrm{mm}$ long, 800 Torr argon arc with the evaporation from the molten copper. They found that the temperature decrease was $1000 \mathrm{~K}$ in a $1 \mathrm{~mm}$ region above the anode compared to the pure argon arc with water-cooled copper anode. Razafinimanana et al [3] showed the result of spectroscopic measurements on a $90 \mathrm{~A}, 18 \mathrm{~mm}$ long, atmospheric argon arc with evaporation from copper anode. They also found that the temperature decrease was $2000 \mathrm{~K}$ near the anode. Furthermore, they showed a density of neutral copper atoms derived from the measurement of line spectrum intensity. Farmer et al [4] showed the results of spectroscopic measurements on a 200 A, $5 \mathrm{~mm}$ long, atmospheric argon arc with evaporation from molten SUS304 which was inserted into copper anode. They reported that the metal vapor had no significant influence on the temperature distribution in the arc. They considered that the strong cathode jet in the case of 200 A of argon arc counteracted any tendency for metal vapor to flow or diffuse from anode to the arc. In computational investigations, Menart and Lin [5] showed the results of numerical calculation on a $200 \mathrm{~A}, 10 \mathrm{~mm}$ long, atmospheric argon arc with copper evaporation. They predicted that the temperature decrease was $2000 \mathrm{~K}$ near the anode. Gonzalez et al [6] calculated atmospheric argon arcs on a 200 or $300 \mathrm{~A}, 10 \mathrm{~mm}$ long with iron evaporation. Effects of metal vapor on transport and radiant properties of the arc were taken into account in their model. They also predicted that the temperature decrease was $2000 \mathrm{~K}$ near the anode.

From the above results, it can be considered that there is a problem in results. It is that both experimental and computational investigations have been carried out under the assumption of Local Thermodynamic Equilibrium (LTE) for the arc. For example, Farmer et al [4] measured temperature distributions of the arc by using the Fowler-Milne method. This technique needs the accurate concentration of metal vapor under the assumption of LTE. However, Farmer et al also needed the temperature for estimating the concentration of metal vapor by using of the spectroscopic analysis. Therefore, they first choose the Fowler-Milne method for temperature measurements under assumption of LTE in pure argon arc plasma, although their arc plasma would be affected by the slight metal vapor. Recently, some of non-LTE states in GTA plasma were showed in case of the pure argon. Snyder et al directly measured temperatures of electron and heavy particle in DC plasma jet [7] and free-burning arc [8] at atmospheric pressure by the method of laser scattering measurement which needs no assumption of LTE. They showed that electron temperature was different from heavy particle temperature. Bentley [9] and Murphy [10] also showed deviation from LTE in GTA plasma. Furthermore, Tanaka and Ushio [11] showed non-LTE state in argon GTA and insisted that the non-LTE state in GTA was caused due to the cathode jet induced by arc constriction at the tungsten electrode. In the case of metal contaminated arc, non-LTE result is also expected. The diagnosis without LTE assumption should be very important for exactly analyzing the metal contaminated arc plasma.

In the present paper, electron temperature measurement of arc during welding is conducted that by using Thomson scattering method without LTE assumption. Furthermore, helium was used as shielding gas. Number density distributions of metal particles were also measured by using the spectroscopic analysis based on more accurate temperature measured by Thomson scattering method. 


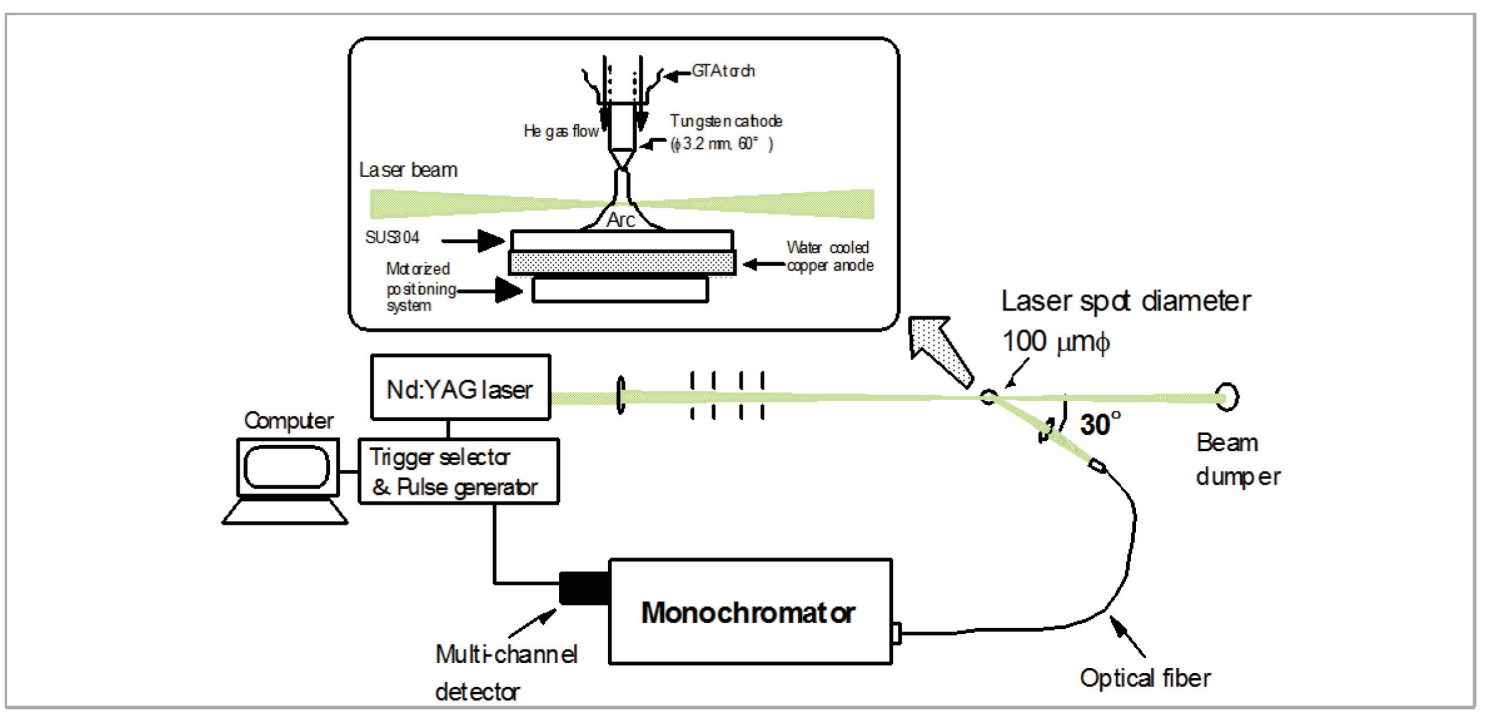

Fig. 1 Schematic illustration of the apparatus for laser scattering measurements.

\section{TEMPERATURE DISTRIBUTIONS}

\subsection{Experimental set up}

In present work, helium gas was used as a shielding gas. This was due to two characteristics of helium gas. One is low arc pressure [12]. This characteristic makes possible for metal vapor to arise more. Thus, it is applicable to investigate effect of metal vapor on the arc plasma. Another one is optically thin characteristic of helium. This characteristic prevents line spectrum absorption in plasma. Thus, this is applicable to observation of metal line spectrum.

Figure 1 shows a schematic illustration of the apparatus for the Thomson scattering measurements. The laser light (wave length: $532 \mathrm{~nm}$, beam waist: 100 $\mu \mathrm{m})$ was focused on the arc plasma and scattered light was detected at the scattering angle $30^{\circ}$. Slit width of monochromator used in the scattering measurement was $300 \mu \mathrm{m}$ and height is $20 \mathrm{~mm}$. Measured scattering signal was the integration of signals for 500 laser pulses. Other details of experimental set up for Thomson scattering is referred to our previous paper [11]. Helium GTA plasma was established between a tungsten cathode with $2 \%$ $\mathrm{La}_{2} \mathrm{O}_{3}$ (diameter $3.2 \mathrm{~mm}$ with tip angle $60^{\circ}$ ) and stainless steel SUS304. The arc gap was fixed at $5 \mathrm{~mm}$, and helium gas flow rate was $401 / \mathrm{min}$. Bead-on-plate welding was conducted by moving SUS304 anode with automatic stage. Welding speed was $3 \mathrm{~cm} / \mathrm{min}$. This speed is too slow compared with that in usual applications. But this slow speed enables for arc to be more stable.

The theory of Thomson scattering is explicated by many literatures $[7-11,13]$.

\subsection{Results and discussion}

Figure 2 shows the comparison between electron temperature for the pure helium arc with the water-cooled copper anode (left side) and that for the helium arc during welding (right side) under the same condition of $150 \mathrm{~A}$ in arc current and $5 \mathrm{~mm}$ in arc length. Figure 2 also shows characteristic appearances of the arc plasma in both cases. In the case of welding, there is a large, wide region of blue luminous plasma in the lower part of the arc. It is supposed that this blue luminous plasma appears to be mainly composed of metal vapor from the weld pool. In the region of $1 \mathrm{~mm} \sim 3 \mathrm{~mm}$ from cathode tip, electron temperatures of both arcs are almost the same. On the other hand, in the region of 3 $\mathrm{mm} \sim 4 \mathrm{~mm}$ from cathode tip, the electron temperature of GTA during welding decreases remarkably compared with the pure helium GTA plasma. Maximum difference of electron temperature reaches about $6000 \mathrm{~K}$ in the arc fringe.

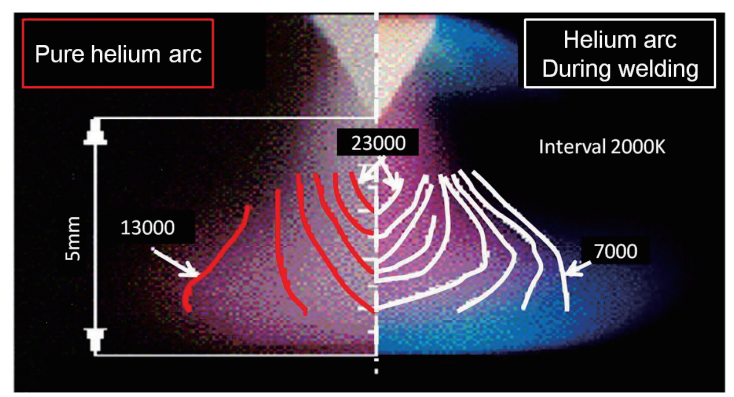

Fig. 2 Comparison between electron temperature for pure helium arc with the water-cooled copper anode (left side) and that for helium arc during welding (right side) under the same condition of $150 \mathrm{~A}$ in arc current and 5 $\mathrm{mm}$ in arc length.

\section{DENSITY DISTRIBUTIONS}

3.1 Experimental set up

Since the region in which electron temperature sharply decreases correspond to the blue luminous region as showed in Fig. 2, it can be supposed that metal vapor generated from the weld pool decreases the electron temperature of arc. Thus, metal vapor density distribution was derived from line spectrum intensity measurement. In the case of this measurement, experimental apparatus is similar to that of Thomson scattering except for the laser source. The detected line spectral intensity was decomposed spatially using Abel 
inversion method [14].

\subsection{Technique for density measurement}

Density of each particle $N$ in the arc plasma during welding was derived by using Boltzmann relation,

$$
N=\frac{\varepsilon \cdot \lambda \cdot \mathrm{Z}\left(T_{e x}\right)}{h \cdot c \cdot A \cdot g \cdot \exp \left(-\frac{E}{k_{B} \cdot T_{e x}}\right) \cdot \beta}
$$

where $\varepsilon$ is the decomposed line spectral intensity, $\lambda$ is the wavelength of spectrum, $T_{e x}$ is the excitation temperature, $\mathrm{Z}\left(T_{e x}\right)$ is the partition function, $A$ is the transition probability, $g$ is the statistical weight of state, $E$ is the energy of quantum state and $\beta$ is the response characteristic of a device used in the present work.

We need information about $T_{e x}$ and $\beta$ for applying relation (1) to density measurements. The excitation temperature can be obtained by using Boltzmann plot method [2]. However, Boltzmann plot method is difficult to give accurate or stable temperature, because estimated temperature is strongly affected by only a slight change in the gradient of data plots [15]. Thus, the application of LTE temperature to relation (1) was proposed. The electron number density measured by Thomson scattering gives LTE temperature through the assumption of ionization equilibrium well known as the Saha equation [16]. Therefore, LTE temperature can be defined as the ionization temperature. It is supposed that ionization temperature is not much different from the excitation temperature, and then the application of LTE temperature would be safe instead of the excitation temperature. However, LTE temperature cannot be calculated through the assumption of ionization equilibrium in the region of reduced temperature in Fig. 2. Because it is expected that this region consists of metal vapor and information about the concentration of metal vapor is nothing. Fortunately, Tanaka and Ushio [11] found that LTE temperature was in accordance with the electron temperature in the region close to the anode. On the other hand, as showed in Fig. 2, the electron temperature in the case of welding arc is in accordance with that in the case of pure helium arc in the distance range of $0 \sim 3 \mathrm{~mm}$ from the cathode tip. This means that LTE temperature in the case of pure helium GTA plasma can be applied to this density measurement only for this distance range. Thus, it can be assumed that the excitation temperature distributions in the helium GTA plasma during welding are as showed in Fig. 3.

Since we already know, excitation temperature, electron temperature, electron densities and helium ion density (that is assumed to be equal to the electron density), number density of neutral helium atom in the case of pure helium plasma at the atmospheric pressure can be easily estimated by using Dalton's partial pressure law. Thus, if a line spectral intensity of helium atom in pure helium plasma is measured, the information about $\beta$ can be got through relation (1).

The information about $T_{e x}$ and $\beta$ of relation (1) can be got as showed above, and the density of each particle in the arc plasma during welding can be estimated by measuring the line spectral intensity of each particle. The wavelengths of each particle measured in present work are showed in Table 1 . The density of helium ion and helium neutral atom was derived from an assumption and a law. The assumption is that sum total of ion density are equal to the electron number density measured by Thomson scattering,

$$
n_{e}=n_{H e \mathrm{II}}+n_{C r \mathrm{II}}+n_{M n \mathrm{II}}+n_{F e \mathrm{II}}
$$

and Dalton's partial pressure law was used, which is represented by

$$
\begin{aligned}
P=\left(n_{\text {HeI }}+n_{C r \mathrm{I}}+\right. & n_{M n \mathrm{I}}+n_{F e \mathrm{I}}+n_{H e l \mathrm{I}}+n_{C r \mathrm{II}} \\
& \left.+n_{M n \mathrm{II}}+n_{F e \mathrm{II}}\right) k_{B} T_{e x}+n_{e} k_{B} T_{e}
\end{aligned}
$$

where, $T_{e}$ is the electron temperature measured by Thomson scattering method.

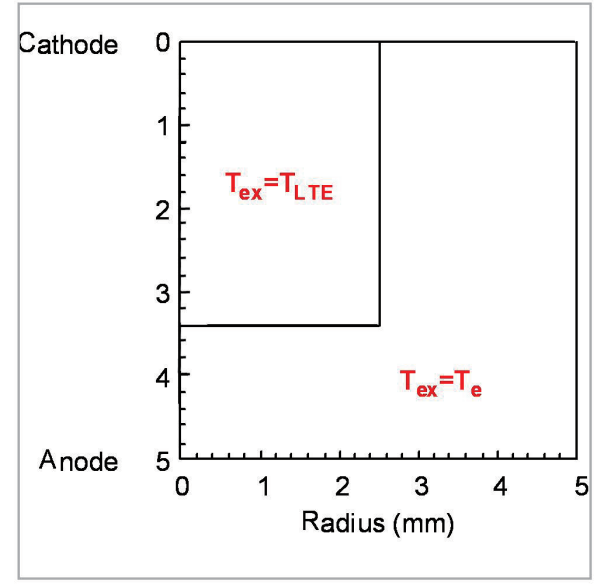

Fig. 3 Excitation temperature distributed in the helium

GTA plasma during welding. It is derived from assuming that LTE temperature is equal to the excitation temperature.

Table 1 Wavelength of metal particle used in spectrum intensity measurements.

\begin{tabular}{|c|c|}
\hline Particle & Wavelength $(\mathrm{nm})$ \\
\hline Iron atom (Fe I ) & 430.790 \\
\hline Iron ion (Fe II ) & 516.900 \\
\hline Chromium atom (Cr I ) & 458.390 \\
\hline Chromium iron (Cr II ) & 455.86 \\
\hline Manganese atom (Mn I ) & 476.153 \\
\hline Manganese iron (Mn II ) & 347.413 \\
\hline
\end{tabular}


3.3 Results and discussion

Figure 4 and 5 show the result of neutral and ion density distributions of helium, iron, chromium, manganese and total metal in the arc axis, respectively. A hatched band is about $3.2 \mathrm{~mm}$ from the cathode. It is suitable for the boundary zone between the blue luminous plasma and another in Fig. 2. The density of helium ion reaches $2.3 \times 10^{22} \mathrm{~m}^{-3}$ near the cathode and decreases by increasing distance from the cathode. On the other hand, each particle of chromium, iron and manganese increases sharply between hatched band and anode. The highest density in the metal particles is chromium. The maximum density reaches $1.6 \times 10^{22} \mathrm{~m}^{-3}$ near the anode. Chromium, manganese and iron are in order of amount. This order should be related to the boiling point, ionization potential of each metal and the chemical compositions of SUS304 used in the present work. It was shown that the plasma states of arc plasma during welding very clearly. Between cathode tip and hatched band, ions in the plasma almost consist of helium only. Between hatched band and anode, contrarily, ions almost consist of metals. Thus, it can be considered that the arc plasma during welding consists of two plasma regions, namely, pure helium plasma region and sort of like quasi-metal plasma region. This change of the plasma states obviously occurs at the hatched band as showed in Fig. 4 and 5. Thus, the quasi-metal plasma region is certainly in good agreement with the electron temperature decrease region in Fig.2. It means that metal vapor contamination significantly decreases the electron temperature in the arc plasma.

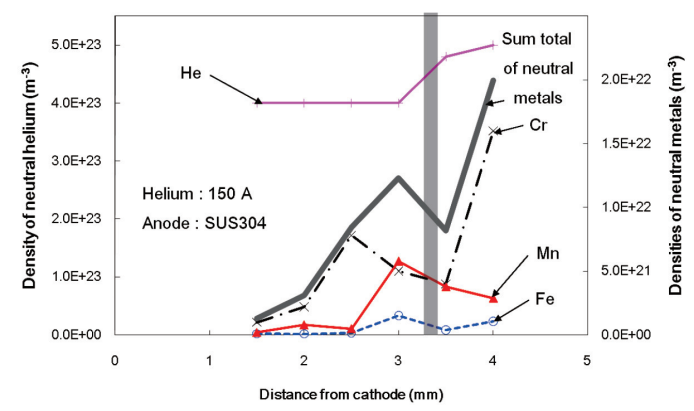

Fig. 4 Dependence of neutral density on distance from cathode.

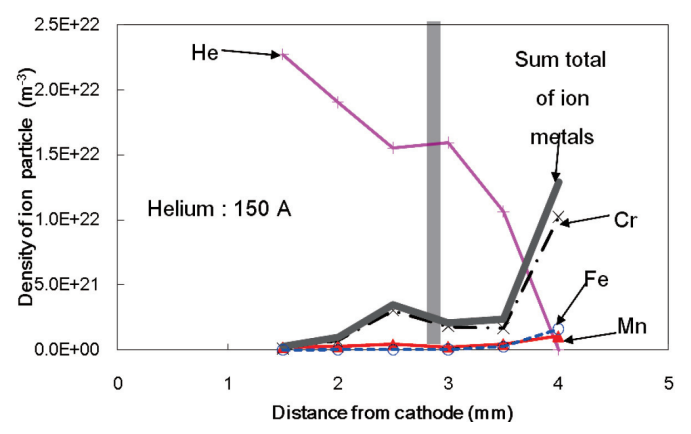

Fig. 5 Dependence of ion density on distance from cathode.

\section{CONCLUSION}

The effects of metal vapor on the plasma state in welding process were investigated. The electron temperatures of helium GTA plasma were measured by Thomson scattering method without LTE assumption. The result showed that the electron temperature of arc during welding sharply decreased near the anode compared with that of pure helium plasma. Furthermore, metal particle density distributions in arc during welding were derived from line spectral intensity measurement. It was showed that the electron temperature decrease region was consistent with quasi-metal plasma region. These results clarified that electron temperature decrease during welding was due to metal contamination and that plasma during welding consisted of two plasma regions, namely, pure helium plasma region and quasi-metal plasma region.

\section{References}

[1] S.S. Glickstein, Weld. J., 55, 222s-229s (1976).

[2] K. Etemadi and E. Pfender, Plasma Chem. Plasma Process., 5, 175-182 (1985).

[3] M. Razafinimanana, et.al., Plasma Sources Sci. Technol., 4, 501-510 (1995).

[4] A.J.D. Farmer, et.al., J. Phys. D: Appl. Phys., 19, 1723-1730 (1986).

[5] J. Menart and L. Lin, Plasma Chem. Plasma Process., 19, 153-170 (1999).

[6] J.J. Gonzalez, et.al., J. Appl. Phys., 74, 3065-3070 (1993).

[7] S.C. Snyder, et.al., Phys. Rev. E, 47, 1996-2005 (1993).

[8] S.C. Snyder, et.al., Phys. Rev. E, 48, 4124-4127 (1993).

[9] R.E. Bentley, J. Phys. D Appl. Phys., 30, 2880-2886 (1997).

[10] A.B. Murphy, et.al., Appl. Phys. Lett., 60, 1304-1306 (1992).

[11] M. Tanaka and M. Ushio, J. Phys. D Appl. Phys., 32, 1153-1162 (1999).

[12] J.F. Lancaster (ed), THE PHYSICS OF WELDING, PERGAMON PRESS, 239 (1984).

[13] T.P. Hughes, Plasma and Laser Light, Adam Hilger, 82-93 (1975).

[14] O.H. Nester and H.N. Olsen, SIAM Rev., 2, 200-207 (1960).

[15] K. Hiraoka, et.al., Quarter J. Jpn Weld. Soc., 14, 641-648 (1996).

[16] M.I. Boulos, et.al., THERMAL PLASMA, 159 (1994).

(Received December 31, 2009; Accepted June 18, 2010) 